\title{
Patterns of Tobacco-Use Behavior Among Chinese Smokers with Medical Conditions
}

\author{
Candice C. Wong · Elisa K. Tong · Janice Y. Tsoh • \\ Meng-Jinn Chen $\cdot$ Fred B. Hom
}

Published online: 26 August 2009

(c) The Author(s) 2009. This article is published with open access at Springerlink.com

\begin{abstract}
Understanding the characteristics of Chinese American smokers with medical conditions and factors associated with their tobacco-use behaviors will guide effective cessation programs. In 2008, the authors described socio-demographic profiles of Chinese smokers with medical conditions treated during the period 2002-2006, documented their tobacco-use behaviors (i.e., average daily cigarette use, nicotine dependence, and number of pastyear quit attempts), and drew comparisons between subjects recruited from hospitals (IP) and ambulatory settings (OP). Compared to OP, IP were significantly older, less educated, less acculturated, and more likely to be retired. Of the two groups, IP had poorer disease profiles, smoked less (4.4 vs. 11.9 cigarettes per day), and had lower nicotine-addiction scores (5.5 vs. 6.7). There was no difference
\end{abstract}

\section{C. Wong $(\bowtie)$}

Institutes for Health and Aging, University of California,

San Francisco, 3333 California Street, Suite 340, San Francisco,

CA 94118-0646, USA

e-mail: Candice.Wong@ucsf.edu

E. K. Tong

Department of Internal Medicine, University of California,

Davis, 4150 V Street, Suite 2400, Sacramento, CA 95817, USA

J. Y. Tsoh

Department of Psychiatry, University of California,

San Francisco, 401 Parnassus Avenue (0984-TRC),

San Francisco, CA 94143, USA

M.-J. Chen

Pacific Institute for Research and Evaluation, 1995 University

Avenue, Suite 450, Berkeley, CA 94704, USA

F. B. Hom

Department of Medicine, University of California,

San Francisco, 3333 California Street, Suite 340,

San Francisco, CA 94118-0646, USA between groups in past-year quit attempts. After adjustments, the data revealed that being employed and OP was associated with higher average daily cigarette use; IP were less nicotine dependent than OP; and for both groups, years of smoking was negatively associated with past-year quit attempts. Our study suggests that, more than acculturation level, health status influences the Chinese smoker's level of cigarette use and nicotine addiction. Given the severity of their disease profiles, IP should be aggressively targeted for intervention, as they are more likely to be light smokers and to be less nicotine dependent than OP. Future tobacco treatment studies should pay attention to health status among smokers in health-care settings in order to provide a more accurate assessment of treatment needs and of barriers to successful smoking cessation.

Keywords Chinese American · Smoking cessation · Health status - Tobacco-use behaviors

\section{Introduction}

Cigarette smoking is one of the leading causes of death in the United States (US) [1]. Among Chinese Americans, cardiovascular disease and cancer account for more than $65 \%$ of all deaths [2]. Despite health concerns associated with cigarette smoking, relatively little is known about tobacco-use behaviors among Chinese American smokers in health-care settings. Former smokers frequently cite health concerns or major illnesses as their reasons for quitting [3, 4]. This finding, coupled with reports that nearly half of Chinese Americans surveyed cited doctors as useful and trusted sources of health information, suggests that health-care settings are potentially important venues 
for providing smoking-cessation treatment and intervention for Chinese smokers [5].

Studies find that shifts in tobacco-use behaviors among Chinese American smokers occur with adaptation to US culture [5-11]. Immigrants are more likely to be current smokers; alternatively, greater acculturation, as measured by English-language proficiency, has been associated with less-frequent smoking among Chinese men [11, 12]. In terms of quit attempts, foreign-born Chinese smokers are less likely to have tried to quit smoking than US born Chinese (59 vs. 69\%) [6]. Acculturation, measured by language preference and length of time in the US, also influences quitting behavior [7]. With the exception of a study that found no difference in health status between former and current smokers [9], population-based studies have failed to examine the effects of ill health on tobacco use among Chinese American smokers.

By some estimates, light smoking (fewer than ten cigarettes per day) has increased by $29 \%$ in California. Light smoking is high among ethnic minorities, including Asian Americans [13]. Available data suggests that health status may affect patterns of tobacco use and quitting behavior, especially for light smokers [14, 15]. Non-daily smokers try to quit more often than daily smokers, and, compared to daily smokers, are more likely to try again within 6 months of a failed attempt [15]. Although non-daily smokers report better health than daily smokers, non-daily smokers report more symptoms of ill health, higher alcohol use, more missed work days, and more visits to hospital emergency rooms than those who have never smoked. The paucity of information about factors affecting cessation in the Chinese American population undermines efforts to guide the delivery of effective cessation programs in health-care settings. Maximizing tobacco control requires an understanding of the specific characteristics of Chinese American smokers and the factors associated with Chinese American tobacco-use behaviors.

The purpose of our study was to describe tobacco-use behaviors among a sample of Chinese American smokers with medical conditions, and to examine the associations between patterns of tobacco use and health status by comparing smokers from hospitals (IP) with those from ambulatory settings (OP). We hypothesized that the number of cigarettes smoked daily would be lower among IP participants, and that IP nicotine-addiction scores and pastyear quit attempts would be higher than those for OP. We concluded that health-care providers need a better understanding of patterns of tobacco-use behaviors among smokers with medical conditions in order to guide their treatment decisions. Better treatment decisions, in turn, increase the likelihood that this vulnerable segment of smokers will successfully quit smoking.

\section{Methods}

The study was part of a baseline assessment from a community-based, culturally tailored, randomized smoking-cessation clinical trial, the Chinese Community Smoking Cessation Project (CCSCP) [16]. From November 2002 to August 2006, smokers were recruited from eight hospitals (one public, one university, two affiliated with a health maintenance organization [HMO], and four private hospitals); six ambulatory clinics (two HMO-affiliated and four community health centers) and through media outreach (e.g., posters and radio announcements in the San Francisco Bay Area). California has a large Asian population (4.2 million); in the San Francisco Bay Area, Chinese are the largest Asian subgroup, comprising $36 \%$ of all local Asian Americans. Recruitment efforts were primarily focused on neighborhoods with a large Chinese American population. All participating clinics and health centers included Chinese-speaking health-care providers.

Eligibility criteria required that participants: (a) were aged 18 years or older; (b) self-identified as Chinese; (c) had smoked tobacco within the past 3 months; (d) spoke Chinese or English; (e) lived within the San Francisco Bay Area; (f) were not currently engaged in assisted smokingcessation efforts; and (g) had a current medical condition. Exclusion criteria were: (a) inability to read and speak English or Chinese; (b) a medically unstable condition; (c) cognitive impairment; and (d) addiction to alcohol or other recreational drugs. A total of 464 participants enrolled in the study.

Physicians and medical records verified the primary diagnoses of participants recruited by research nurses from hospitals and clinics. Participants recruited through media outreach $(N=78)$ reported their own primary diagnoses. When participants had more than 1 medical diagnosis, the principal investigator (a medical doctor) and 3 research staff (a foreign-trained medical doctor, a master level registered nurse, and a nurse-practitioner) determined that the primary diagnosis was that which was most critical and/ or tobacco-related.

Data collection consisted of structured in-person interviews conducted by five trained, bilingual research nurses and/or health educators. The instruments were culturally and linguistically adapted, tested on focus groups, and reviewed by community advisors. The study protocol and consent forms were approved by the Institutional Review Board (IRB), Committee on Human Research, University of California at San Francisco, and by the IRB at each hospital and clinic site. Participants had the option of being interviewed in Mandarin, Cantonese, Toisanese, or English. Participants were paid $\$ 40$ each for interviews. 


\section{Measures}

Socio-demographics. We obtained personal information about participants' age, gender, employment status, education, marital status, household income, birthplace, and indicators of acculturation, including number of years in the US, language preference, and English-language fluency.

Acculturation. To indicate level of acculturation, we calculated a score based on length of stay in the US, language preference, and English fluency. Length of stay was coded as 0 (5 years or less), 1 (6-15 years), and 2 (16 or more years). For language preference, a score of 1 was given if the preferred language was English; other language preferences were scored 0. English language fluency was coded as 0 (not fluent), 1 (somewhat fluent), and 2 (extremely fluent). These variables were totaled to produce a sum score. The resultant acculturation score ranged from 0 (not acculturated) to 5 (extremely acculturated).

Tobacco use. Participants were asked the age at which they smoked their first cigarette, and the age at which they became a regular smoker. They were asked the number of years they had smoked regularly, number of serious attempts they had made to quit in the preceding 12 months, number of cigarettes smoked in the preceding 7 days (prior to hospital admission for participants recruited from hospitals, and prior to baseline assessment for other participants). Average daily cigarette use was determined by dividing the total number of cigarettes smoked by 7 .

Nicotine dependence. The Fagerstrom Test for Nicotine Dependence (FTND) scale was used to measure level of nicotine dependence [17].

Patient status. We categorized smokers recruited from hospitals as "IP"; those recruited from other sources (e.g., clinics, health centers, and by media outreach) were designated "OP".

Primary diagnosis. Participants' primary diagnoses were identified at recruitment; primary diagnoses included cardiovascular disease (CVD) and CVD risk factors (e.g., hypertension, hyperlipidemia), cancer, diabetes, gastrointestinal disease, pulmonary disease, genital-urinary disease, and other illnesses.

\section{Data Analyses}

Descriptive statistics were presented to summarize participants' socio-demographic profiles, primary diagnoses, and patterns of tobacco use. A major hypothesis of this study was that IP and OP might differ in smoking behaviors due to differences in health status; therefore, we also compared IP and OP profiles and patterns of tobacco use. ANOVA analyses examined the means and $\chi^{2}$ tests. Multiple regression analyses were then performed to assess the associations of socio-demographic variables, acculturation, and years of smoking with tobacco-use variables (i.e., average daily cigarette use, level of nicotine dependence, and past-year quit attempts), and, finally, with IP or OP status. In the analyses, IP or OP patient status was used as a proxy for participants' health: IP implied poorer health than OP. We conducted two-step multiple regression analyses. The step-one model included age, education, employment, marital status, acculturation, and years of smoking as predictors of tobacco-use variables. The step-two model included in- or out- patient status. Dichotomous variables were generated for education level, employment, marital status, and patient status. Gender was not included in the analyses as a variable because more than $90 \%$ of the participants were men. Missing or incomplete data precluded our use of household income as a variable. All of these analyses were conducted using Microsoft Windows' Statistical Package for Social Sciences (SPSS) 15.0 [18].

\section{Results}

\section{Sample Characteristics}

Two-thirds of the participants were recruited from ambulatory settings. Details of socio-demographics for the study sample are presented in Table 1 . The study sample was predominately male. Their ages ranged from 18 to 89 years, with a mean age of 58.3. About four-fifths were married or lived with a partner. About one-third were high school graduates. Two-fifths were employed. One-half reported an annual household income of less than $\$ 20,000$. The vast majority $(73 \%)$ was born in mainland China. More than half had resided in the US 16 years or longer. Most (86\%) preferred to communicate in Cantonese, and only $10 \%$ reported fluency in English.

The demographic profiles of participants recruited from in- and outpatient settings differed in many ways (see Table 1). Compared to IP, OP were younger, more likely to be employed, had more years of formal education, reported higher household income, and reported greater English fluency (this last resulted in higher acculturation scores). In contrast, the majority of IP had less than a high school education (78 vs. 46\%) and spoke no English (65 vs. 28\%).

\section{Primary Diagnosis}

As shown in Table 2, over 75\% of IPs' diagnoses were tobacco-related. Among IPs with CVD diagnoses, 65\% were admitted with coronary artery disease or stroke. In contrast, only $8 \%$ of OP had a CVD diagnosis; $41 \%$ had CVD risk factors such as hypertension or hyperlipidemia. 
Table 1 Socio-demographic profile of Chinese smokers with medical conditions

\begin{tabular}{|c|c|c|c|c|c|c|c|}
\hline & \multicolumn{2}{|c|}{ Total $(N=460)$} & \multicolumn{2}{|c|}{ Inpatients $(n=175)$} & \multicolumn{2}{|c|}{ Outpatients $(n=289)$} & \multirow[t]{2}{*}{$P$} \\
\hline & $N$ & $\%$ & $N$ & $\%$ & $N$ & $\%$ & \\
\hline Gender & & & & & & & 0.312 \\
\hline Female & 40 & 8.6 & 12 & 6.9 & 28 & 9.7 & \\
\hline Male & 424 & 91.4 & 163 & 93.1 & 261 & 90.3 & \\
\hline Age & & & & & & & 0.000 \\
\hline$\leq 50$ & 134 & 28.9 & 21 & 12.0 & 113 & 39.1 & \\
\hline $51-64$ & 141 & 30.4 & 35 & 20.0 & 106 & 36.7 & \\
\hline $65-74$ & 128 & 27.6 & 70 & 40.0 & 58 & 20.1 & \\
\hline$\geq 75$ & 61 & 13.1 & 49 & 28.0 & 12 & 4.2 & \\
\hline Marital status & & & & & & & 0.342 \\
\hline Married/partnered & 363 & 78.3 & 141 & 80.6 & 222 & 76.8 & \\
\hline Single/divorced/widowed & 101 & 21.8 & 34 & 19.4 & 67 & 23.2 & \\
\hline Education & & & & & & & 0.000 \\
\hline Less than high school & 269 & 57.9 & 136 & 77.7 & 133 & 46.0 & \\
\hline High school/trade/some college & 142 & 30.6 & 27 & 15.4 & 115 & 39.8 & \\
\hline Bachelor/master/doctorate & 53 & 11.4 & 12 & 6.9 & 41 & 14.2 & \\
\hline Employment status & & & & & & & 0.000 \\
\hline Full time/part time & 191 & 41.1 & 40 & 22.9 & 151 & 52.2 & \\
\hline Unemployed & 58 & 12.5 & 13 & 7.4 & 45 & 15.6 & \\
\hline Retired/other & 215 & 46.3 & 122 & 69.7 & 93 & 32.2 & \\
\hline Household income $^{a}$ & & & & & & & 0.000 \\
\hline$<\$ 19,000$ & 235 & 50.6 & 112 & 82.4 & 123 & 50.4 & \\
\hline$\$ 20-100,000+$ & 145 & 31.3 & 24 & 17.6 & 121 & 49.6 & \\
\hline Birthplace & & & & & & & 0.000 \\
\hline United States & 24 & 5.2 & 2 & 1.1 & 22 & 7.6 & \\
\hline Hong Kong & 49 & 10.6 & 9 & 5.1 & 40 & 13.8 & \\
\hline Mainland China & 342 & 73.7 & 153 & 87.4 & 189 & 65.4 & \\
\hline Other & 49 & 10.6 & 11 & 6.3 & 38 & 13.1 & \\
\hline Years of stay in the US & & & & & & & 0.171 \\
\hline$\leq 5$ years & 57 & 12.3 & 15 & 8.6 & 42 & 14.5 & \\
\hline $6-15$ years & 134 & 28.9 & 52 & 29.9 & 82 & 28.4 & \\
\hline 16 or more & 272 & 58.6 & 107 & 61.5 & 165 & 57.1 & \\
\hline Language preference & & & & & & & 0.021 \\
\hline English & 31 & 6.7 & 5 & 2.9 & 26 & 9.0 & \\
\hline Cantonese & 400 & 86.2 & 161 & 92.0 & 239 & 82.7 & \\
\hline Mandarin & 29 & 6.3 & 7 & 4.0 & 22 & 7.6 & \\
\hline Other & 4 & 0.9 & 2 & 1.1 & 2 & 0.7 & \\
\hline English fluency & & & & & & & 0.000 \\
\hline Not at all & 195 & 42.0 & 113 & 64.6 & 82 & 28.4 & \\
\hline Somewhat/moderately & 222 & 47.8 & 55 & 31.4 & 167 & 57.8 & \\
\hline Very well/extremely well & 47 & 10.1 & 7 & 4.0 & 40 & 13.8 & \\
\hline Acculturation score & & & & & & & 0.000 \\
\hline 0 to $<2$ & 119 & 25.6 & 52 & 29.7 & 67 & 23.2 & \\
\hline 2 to $<4$ & 306 & 66.0 & 116 & 66.3 & 190 & 65.7 & \\
\hline 4 to $<5$ & 39 & 8.4 & 7 & 4.0 & 32 & 11.1 & \\
\hline
\end{tabular}

a 84 cases with missing data (e.g., declined to state and do not know) were excluded from analyses. These cases were equally distributed between in- and out-patients

Bold values indicate significant values at $p<0.05$ 
Table 2 Primary diagnoses of smokers with medical conditions

\begin{tabular}{|c|c|c|c|c|c|c|}
\hline \multirow[t]{2}{*}{ Diagnosis } & \multicolumn{2}{|c|}{ Inpatients $(n=175)$} & \multicolumn{2}{|c|}{ Outpatients $(n=289)$} & \multicolumn{2}{|c|}{ Total $(N=460)$} \\
\hline & $N$ & $\%$ & $N$ & $\%$ & $N$ & $\%$ \\
\hline \multicolumn{7}{|l|}{ Cardiovascular disease } \\
\hline CVD risk factors (e.g., hypertension) & - & - & 118 & 40.8 & 118 & 25.4 \\
\hline Coronary artery disease & 42 & 24.0 & 22 & 7.6 & 72 & 13.8 \\
\hline Stroke/TIA & 6 & 3.4 & 2 & 0.7 & 8 & 1.7 \\
\hline Arrhythmias & 3 & 1.7 & 0 & 0.0 & 3 & 0.6 \\
\hline \multicolumn{7}{|l|}{ Pulmonary disease } \\
\hline COPD/emphysema & 19 & 10.9 & 7 & 2.4 & 26 & 5.6 \\
\hline Pneumonia/pleural effusion & 31 & 18.9 & 1 & 0.3 & 30 & 7.4 \\
\hline Bronchitis/asthma & 4 & 2.3 & 25 & 8.7 & 29 & 6.2 \\
\hline Pneumothorax & 6 & 3.4 & 1 & 0.3 & 7 & 1.5 \\
\hline Other (e.g., TB, thoracotomy) & 6 & 3.1 & 9 & 3.4 & 15 & 3.1 \\
\hline Cancer & 12 & 6.9 & 6 & 2.1 & 18 & 3.9 \\
\hline Diabetes & 4 & 2.3 & 26 & 9.0 & 30 & 6.5 \\
\hline Gastrointestinal disease & 31 & 17.7 & 9 & 3.1 & 40 & 8.6 \\
\hline Genital-urinary disease & 11 & 6.3 & 8 & 2.8 & 19 & 4.1 \\
\hline Other (e.g., skin disorder) & - & - & 52 & 18.0 & 52 & 11.2 \\
\hline
\end{tabular}

Table 3 Tobacco-use behavior and nicotine dependence among Chinese smokers with medical conditions

\begin{tabular}{|c|c|c|c|}
\hline & $\begin{array}{l}\text { Inpatients }(n=175) \\
M(\mathrm{SD})\end{array}$ & $\begin{array}{l}\text { Outpatients }(n=289) \\
M(\mathrm{SD})\end{array}$ & $P$ \\
\hline Age of first cigarette & $17.8(7.7)$ & $18.3(6.0)$ & 0.438 \\
\hline Years of smoking regularly & $47.5(16.7)$ & $33.2(14.3)$ & 0.000 \\
\hline Average number of cigarettes used per day & $4.4(5.6)$ & $11.9(8.0)$ & 0.000 \\
\hline Number of serious quit attempt in past year & $1.1(2.4)$ & $1.5(2.6)$ & 0.077 \\
\hline \multirow[t]{2}{*}{ Nicotine dependence score } & $5.5(1.7)$ & $6.7(2.0)$ & 0.000 \\
\hline & $\begin{array}{l}\text { Inpatients }(n=175) \\
N(\%)\end{array}$ & $\begin{array}{l}\text { Outpatients }(n=289) \\
N(\%)\end{array}$ & $P$ \\
\hline Tobacco use category (cigs/day) & & & 0.000 \\
\hline$\leq 5$ & $119(68.0)$ & $64(22.1)$ & \\
\hline $6-10$ & 33 (18.9) & $96(33.2)$ & \\
\hline $11-19$ & $13(7.4)$ & $52(18.0)$ & \\
\hline$\geq 20$ & $10(5.7)$ & 77 (26.6) & \\
\hline \multicolumn{4}{|l|}{ Nicotine dependence items } \\
\hline Hate to give up the first cigarette of the day & $82(46.9)$ & $172(59.5)$ & 0.008 \\
\hline Smoke more in the morning & $89(50.9)$ & $161(55.7)$ & 0.310 \\
\hline Smoke when ill & $21(12.0)$ & $70(24.2)$ & 0.001 \\
\hline Difficult refraining from smoking & $35(20.0)$ & $77(26.6)$ & 0.105 \\
\hline First smoke of the day from awakening (min) & & & 0.342 \\
\hline$\leq 5$ & $57(32.6)$ & $107(37.0)$ & \\
\hline $6-30$ & $49(28.0)$ & $90(31.1)$ & \\
\hline $31-60$ & $29(16.6)$ & $44(15.2)$ & \\
\hline$>60$ & $40(22.9)$ & $48(16.6)$ & \\
\hline
\end{tabular}

Bold values indicate significant values at $p<0.05$ 
Table 4 Multiple regression analyses assessing tobacco-use behavior among Chinese smokers with medical conditions

\begin{tabular}{|c|c|c|c|}
\hline Predictors & $\begin{array}{l}\text { Average daily } \\
\text { cigarette use }\end{array}$ & $\begin{array}{l}\text { Nicotine } \\
\text { dependence }\end{array}$ & $\begin{array}{l}\text { Past-year } \\
\text { quit attempts }\end{array}$ \\
\hline \multicolumn{4}{|l|}{ Step 1 model } \\
\hline Age & -0.157 & -0.191 & 0.102 \\
\hline Education $(<$ high school $=1)$ & 0.023 & 0.035 & 0.001 \\
\hline Employment $($ employed $=1)$ & $0.223 * * *$ & 0.091 & 0.043 \\
\hline Marital status (partnered $=1$ ) & -0.054 & -0.063 & -0.011 \\
\hline Acculturation score & $0.116^{*}$ & -0.001 & 0.069 \\
\hline Years of smoking & 0.053 & 0.157 & $-0.278^{*}$ \\
\hline \multicolumn{4}{|l|}{ Step 2 model } \\
\hline Age & -0.063 & -0.119 & 0.097 \\
\hline Education $(<$ high school $=1)$ & 0.079 & 0.078 & -0.002 \\
\hline Employment $($ employed = 1) & $0.205 * * *$ & 0.077 & -0.044 \\
\hline Marital status (partnered $=1$ ) & -0.077 & -0.080 & -0.010 \\
\hline Acculturation score & 0.079 & -0.029 & 0.071 \\
\hline Years of smoking & 0.110 & 0.200 & $-0.280 *$ \\
\hline Patient status (inpatient $=1$ ) & $-0.414 * * *$ & $-0.318 * * *$ & 0.022 \\
\hline
\end{tabular}

Note. Cell numbers are standardized regression coefficients

$* P<0.05$

$* * * P<0.001$

Overall, IP appeared to have poorer disease profiles than OP (e.g., CVD and cancer), whereas OP were likely to exhibit risk factors for chronic diseases (e.g., hypertension, hyperlipidemia).

\section{Patterns of Tobacco Use}

Patterns of tobacco use were compared for IP and OP; these results are summarized in Table 3. The two groups did not differ in onset of cigarette use. On average, IP had smoked regularly for a longer period of time than OP, probably because IP were generally older. IP, however, were currently smoking less than OP. They smoked about 4.4 cigarettes per day, compared to 11.9 cigarettes per day among OP. A majority of IP (68\%), smoked 5 or fewer cigarettes on average per day, compared to OP (only $22 \%$ of OP smoked an average of 5 or fewer cigarettes per day). More than 1 in 4 OP $(26.6 \%)$ reported an average daily use of 20 cigarettes or more, compared to about 1 in $20(5.6 \%)$ for IP. There was no significant difference between the two groups in terms of the number of serious quit attempts in the past year.

IP, on average, scored lower FTND nicotine-dependence scores than OP (Table 3). Further exploration of the scale findings indicate that the differences between the 2 groups centered on 2 items. Proportionally, more OP than IP reported that they hated to give up the first cigarette of the day, and they reported smoking even when they were ill.
Factors Associated with Tobacco Use

Average daily cigarette use was significantly and positively predicted by employment status and higher acculturation in the first model (Table 4). When patient status was entered in the model, acculturation was no longer a significant predictor. Overall, being employed was associated with increased average daily cigarette use, and IP was negatively associated with average daily cigarette use.

None of the variables significantly predicted nicotine dependence in the first model. When patient status was entered in the model, being IP was negatively associated with nicotine dependence.

Years of smoking significantly predicted past-year quit attempts. In other words, the longer participants had smoked, the less likely they were to have attempted to quit in the preceding year. Patient status was not significantly associated with past-year quit attempts.

\section{Discussion}

This is the first study to report on tobacco-use behaviors among Chinese American smokers with medical conditions. Our study suggests that the smoker's general state of health, more than the level of acculturation, influences levels of cigarette use and nicotine addiction. On average, IP had smoked fewer daily cigarettes in the past week than OP (4.4 vs. 11.9). IP were also less likely than OP to smoke 
when ill (12 vs. 24\%), and IP had lower nicotine-dependence scores than OP (5.5 vs. 6.7).

In contrast to earlier population-based studies of Chinese smokers $[8,10]$, we did not find a significant association between acculturation, age, and tobacco-use behaviors after adjustment for health status. Instead, our study suggests that individual tobacco-use behavior may largely be due to illness burdens among Chinese smokers with medical conditions. One intriguing finding is that being employed was significantly associated with higher average daily cigarette use, regardless of health status. The majority of employed participants $(60 \%)$ worked in nonskilled jobs, such as restaurant or construction work. It is possible that stress associated with unskilled labor, coupled with workplaces that are likely to tolerate cigarette use, contributed to the higher average daily cigarette use found among this segment of smokers.

The rate of past-year quit attempts among our sample of smokers with medical conditions was considerably lower than rates reported by Chinese immigrants in the California Chinese American Tobacco Survey (CCATUS; 40 vs. 60\%) [7]. The lower rate of past-year quit attempts among our study participants compared to that of CCATUS smokers is surprising, given that smokers with illness burdens are more likely to attempt to quit than "healthier" smokers [4]. One possible reason for this unexpected finding is that a substantial percentage of smokers in our sample were light smokers (e.g., $\sim 40 \%$ smoked $<5$ cigarettes per day, and $68 \%$ smoked $<10$ cigarettes per day), compared to current smokers in the CCATUS study, only $25.8 \%$ of whom reported smoking 5 or fewer cigarettes per day [7]. In our sample, smokers with medical conditions may have already reduced their cigarette use in prior quit attempts. It is plausible that these light smokers did not believe that further reduction or complete cessation of smoking would produce health benefits. For example, almost 50\% of the current smokers in CCATUS endorsed the statement that smoking 5 cigarettes per day has the same health risk as not smoking at all (H Tang, University of California, San Diego, personal communication, May 21, 2008). Therefore, focused education may be necessary to increase the cessation rate, especially among light smokers in the Chinese community.

In light of the fact that the quit-attempt rate has been reported to be the single most significant factor in annual cessation rates across Chinese populations [6], the lower rate of quit attempts in our study of smokers with medical conditions underscores an urgent need for effective cessation programs in health-care settings. Asian Americans are more likely than whites to be non-daily smokers $[19,20]$. In addition, according to a study that controlled for selfreported health status, light smokers and non-daily smokers are more likely to want to quit than daily smokers [14, 15]. In our study, close to 1 in 4 smokers (24\%) reported not smoking on the interview date or on the day prior to hospital admission. The relatively high numbers of non-daily smokers and light smokers among these smokers with medical conditions suggests that clinicians can play a critical role in identification and assessment of smokers in health-care settings, as well as in offering treatment to smokers in those settings. In particular, IP smokers-who were more likely than OP to be light smokers and less nicotine dependent-should be aggressively targeted for intervention. Given the severity of their disease profiles, hospitalization provides an opportune moment to advise IP smokers to quit. However, some clinicians may perceive smokers with worse illness burdens as having greater nicotine dependence, as not being receptive to cessation advice, and as having a lower probability for cessation success; thus some clinicians may be less inclined to provide appropriate intervention. In addition, recent data suggests that non-daily smokers were asked or advised about smoking cessation less often than daily smokers [15]. Thus, clinicians may incorrectly assume that smokers who consume five or fewer cigarettes per day are not addicted to nicotine, especially when these light smokers do not exhibit withdrawal symptoms during periods of abstention, such as hospitalization. Our study suggests that it is critical to inform clinicians that they are important facilitators of smoking cessation, and that they have a prime opportunity to improve the health of their patients who smoke.

Our sample of smokers was recruited primarily from health-care settings and composed of older immigrant men who spoke no English and had low educational levels. Many were retired and only about $40 \%$ were employed, either part- or full-time. As a result, more than half of participants reported annual household income of less than $\$ 20,000$. This particular combination of socio-demographic characteristics (older age, poor, less education, and low English proficiency) suggests that our participants would likely encounter tremendous barriers in seeking appropriate and affordable smoking cessation treatment services. Our study confirms large gaps in tobacco treatment services in the San Francisco Bay Area for Chinese-speaking smokers. During the study period, smoking cessation treatment programs for Chinese speakers were not available at any of the participating hospital sites. Although one HMO offered a smoking cessation group counseling program for outpatients, the program was not accessible to Chinese-speakers. Moreover, the seriousness of medical conditions, particularly among IP smokers (with $>75 \%$ tobacco-related illnesses), and the long duration of their tobacco use (an average of 39 years), underscores the urgent need for timely cessation interventions, particularly in health-care facilities located in or near Chinese neighborhoods.

Several limitations of this study should be noted. Because of the study's selection criteria, the sample population could 
differ from other Chinese American smokers with respect to socio-demographic characteristics and health status. For example, the participants in this study were not randomly selected. Rather, smokers with medical conditions were recruited from hospitals and clinics located in or near Chinese neighborhoods. This selection criterion may bias our results, producing not only a sicker and older population, but also a population with ready access to health care. Participants' characteristics such as education, income, acculturation, and health needs may be different from smokers in other settings. The participants' willingness to quit smoking was another criterion used to enroll smokers into the study. Thus, our sample population could differ from the general population, in which some smokers have little or no interest in quitting. In addition, the study relied on self-reported smoking behavior, and the self-reported medical diagnoses of about $20 \%$ of the smokers recruited through media outreach were not verified. Both factors may limit the study's validity. However, we consider these to be minor limitations, given that prior studies comparing self-reported medical history with verified medical records found substantial agreement (>90\% sensitivity and specificity), particularly for conditions related to cardiovascular diseases and diabetes [21, 22]. Lastly, our sample of smokers included a small number of women $(\sim 9 \%)$. It should be noted that a low smoking prevalence $(<5 \%)$ has been reported among Chinese women in the US [7, 9]. Thus, the findings from CCSCP may not be generalizable to the entire population of Chinese American smokers.

Notwithstanding these limitations, the findings from this study point to both a critical need and tremendous opportunities to implement effective language- and culture-specific smoking-cessation interventions in health-care settings. Future tobacco treatment studies should pay particular attention to health status and illness burden among smokers in order to provide a more accurate assessment of needs, such as elucidating barriers to smoking-cessation treatment in health-care settings.

Open Access This article is distributed under the terms of the Creative Commons Attribution Noncommercial License which permits any noncommercial use, distribution, and reproduction in any medium, provided the original author(s) and source are credited.

\section{References}

1. Novello, A. C. (1993). From the surgeon general, US public health service. Tobacco control. Journal of the American Medical Association, 270, 806.

2. Hoyert, D. L., Kochanek, K. D., \& Murphy, S. (1999). Deaths: Final data for 1997. National Vital Statistics Report, 47, 1-104.

3. McCaul, K. D., Hockemeyer, J. R., Johnson, R. J., Zetocha, K., Quinlan, K., \& Glasgow, R. E. (2006). Motivation to quit using cigarettes: A review. Addictive Behavior, 31, 42-56.
4. Vangeli, E., \& West, R. (2008). Sociodemographic differences in triggers to quit smoking: Findings from a national survey. Tobacco Control. Published online 10 Sep 2008 [http://tobaccocontrol.bmj. com/cgi/content/abstract/tc.2008.025650v1].

5. Ferketich, A. K., Wewers, M. E., Kwong, K., et al. (2004). Smoking cessation interventions among Chinese Americans: The role of families, physicians and the media. Nicotine \& Tobacco Research, 6, 241-248.

6. Zhu, S., Wong, S., Tang, H., Shi, C., \& Chen, M. S. (2007). High quit ratio among Asian immigrants in California: Implications for population tobacco cessation. Nicotine \& Tobacco Research, 9, $505-514$.

7. Carr, K., Beers, M., Kassebaum, T., \& Chen, M. S. (2005). California Chinese American tobacco use survey-2004. Sacramento, CA: California Department of Health Services.

8. Ma, G. X., Shive, S., Tan, Y., \& Toubbeh, J. I. (2002). Prevalence and predictors of smoking behaviors among Asian Americans in the Delaware Valley region. American Journal of Public Health, 92, 1013-1020.

9. Shelley, D., Fahs, M., Scheinmann, R., Swain, S., Qu, J., \& Burton, D. (2004). Acculturation and tobacco use among Chinese Americans. American Journal of Public Health, 94, 300-307.

10. Yu, C. E., Chen, E., Kim, K., \& Abdulrahim, S. (2002). Smoking among Chinese Americans: Behavior, knowledge and beliefs. American Journal of Public Health, 92, 1007-1012.

11. Tang, H., Shimizu, R., \& Chen, M. J. (2005). English language proficiency and smoking prevalence among California's Asian Americans. Cancer, 104, 2982-2988.

12. Fu, S. S., Ma, G. X., Tu, X. M., Siu, P. T., \& Metlay, F. P. (2003). Cigarette smoking among Chinese Americans and the influence of linguistic acculturation. Nicotine \& Tobacco Research, 5, 803811.

13. Gilpin, E., White, M., White, V., et al. (2003). Tobacco control successes in California: A focus on young people, results from the California tobacco surveys, 1990-2002. La Jolla, CA: University of California.

14. Tong, E., Nguyen, T., Vittinghoff, E., \& Perez-Stable, E. J. (2009). Light and intermittent smoking among California's Asian Americans. Nicotine \& Tobacco Research (in press).

15. Tong, E. K., Ong, M. K., Vittinghoff, E., \& Perez-Stable, E. J. (2006). Nondaily smokers should be asked and advised to quit. American Journal of Preventive Medicine, 30, 23-30.

16. Wong, C., Tsoh, J., Tong, E., Cooper, B., Hom, F., \& Chow, E. (2008). The Chinese community smoking cessation project: A community sensitive intervention trial. Journal of Community Health [Epub ahead of print].

17. Heatherton, T. F., Kozlowski, L. T., Frecker, R. C., \& Fagerstrom, K. O. (1991). The fagerstrom test for nicotine dependence: a revision of the fagerstrom tolerance questionnaire. British Journal of Addiction, 86, 1119-1127.

18. SPSS. (1989-2006). Microsoft windows, statistical package for social sciences 15.0. Chicago, IL: SPSS.

19. Hassmiller, K. M., Warner, K. E., Mendez, D., Levy, D. T., \& Romano, E. (2003). Nondaily smokers: Who are they? American Journal of Public Health, 93, 1321-1327.

20. Wortley, P. M., Husten, C. G., Trosclair, A., Chrismon, J., \& Pederson, L. L. (2003). Nondaily smokers: A descriptive analysis. Nicotine \& Tobacco Research, 5, 755-759.

21. Kehoe, R., Wu, S. Y., Leske, C., \& Chylack, L. T. (1994). Comparing self-reported and physician-reported medical history. American Journal of Epidemiology, 139, 813-818.

22. Bush, T. L., Miller, S. R., Golden, A. L., \& Hale, W. E. (1989). Self-report and medical record report agreement of selected medical conditions in the elderly. American Journal of Public Health, 79, 1554-1556. 